\title{
Periodismo político Algo más que periodistas de política
}

\author{
Alicia Miranda Duke
}

Maestría en Ciencia Política

de la UCA, San Salvador

RESUMEN: En este artículo se describen las características individuales y algunas actitudes de los periodistas de política de cuatro medios impresos. El objetivo es conocer la actitud que los periodistas muestran respecto al papel que consideran que tienen en la sociedad, en relación a la información y al trato con la fuente política. Se trata de un grupo que se ve a sí mismo como los garantes de brindar la mejor información posible para tomar mejores decisiones. En ese sentido, aseguran que la profesión periodística tiene una fuerte carga de responsabilidad social. Sin embargo, este compromiso tiene límites en cosas más sustanciales para los periodistas entrevistados: el bajo salario que reciben.

ABSTRACT: The author describes the individual characteristics and some attitudes of the journalists of the politic section from four newspapers. The aim is to know the attitude of the journalists toward the role they consider they have in the society, regarding the information and their relation with the politic sources. It is a group that conceives itself as responsible to bring the best information available to make the best decisions. In this sense, they claim that the journalistic profession has a strong social responsibility. However, this commitment has some burdens in very important matters for the journalists that were interviewed by the author: the low wages they have. 
ada nota publicada es sólo el resultado de un largo proceso en el que los periodistas juegan un rol fundamental. Durante la labor periodística, además de los criterios puramente profesionales también pueden mediar las creencias, percepciones y actitudes de parte de los comunicadores. Por ejemplo, la actitud de los periodistas de política respecto al papel que consideran que tienen en la sociedad.

Si bien se trata de un estudio que no puede verse por separado del proceso que implica la producción periodística, si es posible acercar la lupa a los comunicadores que actualmente están mediando entre el quehacer político y la sociedad. ¿Quiénes son los periodistas de política? ¿Cómo perciben la labor periodística? ¿Qué nivel de satisfacción muestran? Y por otro lado, ¿qué actitudes manifiestan respecto a la información y hacia la fuente política?

En el presente artículo vamos a describir a los periodistas de política de cuatro medios de comunicación impresos. Como veremos en detalle más adelante, se trata de reporteros que en su mayoría ven el trabajo periodístico como algo circunstancial en sus vidas; una actitud que tiene su talón de Aquiles en el salario que reciben. Por otro lado, los reporteros de política reconocen que necesitan de la fuente política, por lo tanto, se muestran a favor de mantener una relación de beneficio mutuo. Incluso, hay quienes hablan de "favorcitos" a la fuente, siempre y cuando no comprometa su labor.

Estas y otras características que se describirán a lo largo del artículo nos ofrecen un marco de referencia para acercarnos al quehacer del periodismo político en El Salvador.

\section{Los periodistas y las mediaciones}

$\mathrm{E}$ n la actualidad, los medios de comunicación mantienen un doble rol. Por un lado, actúan y apelan a ser defensores de los intereses de la sociedad frente al poder, especialmente el político. Por otro, mantienen una relación de beneficio mutuo precisamente con ese poder. Esta duplicidad surge a partir de la teoría de la responsabilidad social', que supone que la prensa goza de las libertades del sistema económico, pero al mismo tiempo le permite arrogarse el papel protector frente a los abusos de los gobernantes.

Una de las exigencias de la teoría de la responsabilidad social es que la función que desempeñan los medios debe realizarse sobre la base de criterios profesionales. "En este sentido, no extraña que se pida, en 
primer lugar, mayor profesionalidad a quienes tienen en sus manos la importante tarea de informar" (Cosa y Peñalva, 1998). Bajo esta lógi$\mathrm{ca}$, los periodistas juegan un papel central pues son los que en primera instancia seleccionan, jerarquizan e interpretan la información para luego publicarla. Es decir, son los primeros en decidir qué hecho es importante que los lectores conozcan. Pero, ¿qué consideran importante? ¿Cuál creen que es su papel en la sociedad? ¿Influye esta actitud en su producción periodística?

Un nivel de análisis para responder a estas preguntas es conocer algunas mediaciones que podrían intervenir durante la producción periodista. Shoemker (Canel, 1999) propuso niveles de análisis que incluyen las características individuales de los periodistas, las rutinas profesionales de los medios, la organización de la redacción, la relación con las fuentes y la competencia interna y externa, entre otros. Estos componentes pueden mediar, voluntaria o involuntariamente, en la elaboración de una nota periodística.

En el 2003, Javier Urrea Cuellar, politólogo de la Pontificia Universidad Javeriana de Colombia, publicó un artículo sobre la realización de noticias en El Salvador ${ }^{2}$ en el que se describen las rutinas que intervienen en la construcción de reportajes en tres medios. En la investigación se ponderó la importancia que las rutinas, como la selección de temas, tienen en la producción periodística.

En la misma línea, en 1950 David Manning White aplicó al periodismo el término "gatekeeping" para explicar que la elaboración de la noticia es subjetiva y que depende de las experiencias, características, actitudes y expectativas del periodista.

El gatekeeping ha sido criticado por considerar únicamente un nivel de análisis individual sustentado en la sociología funcionalista y por no tener en cuenta a la sociedad como caja de resonancia, en términos de intereses y expectativas. Sin embargo, es un modelo que permite describir a los que, como se dijo antes, en primera instancia escogen lo que consideran importante en el quehacer político y lo hacen público. Asimismo, es una perspectiva que ofrece un nivel de análisis necesario para quienes deseen profundizar en el quehacer periodístico como la construcción social de la realidad.

En el presente artículo se tomó este modelo para describir a los periodistas de política y algunas de sus actitudes.

\section{Actitudes}

- xisten muchas definiciones del término actitud, desde Thurstone, que en 1928 la sinteti- zó como "la intensidad de afecto a favor o en contra de un objeto psicológico", hasta otras un poco más 
elaboradas que definen la actitud "como una organización duradera de creencias y cogniciones en general, dotada de una carga afectiva a favor o en contra de un objeto social definido, que predispone a una acción coherente con las cogniciones y efectos relativos a dicho objeto" (Rodrigues, 1980). Pero cualquiera que sea la definición que se adopte, el término conlleva siempre algunas características.

En primer lugar, se entiende a "la actitud como algo que media e interviene entre los aspectos del ambiente externo, que son los estímulos, y las reacciones de la persona, es decir, sus respuestas evaluativas manifiestas" (Morales, 1999). Se trata de un proceso interno que se manifiesta a través de respuestas evaluativas.

En segundo lugar, una actitud siempre se refiere a un objeto. Por ejemplo, un estudio publicado en España en $1999^{3}$ concluyó que los periodistas españoles tienden a mostrar una actitud de abogado; en este caso, el objeto de la actitud es el papel de los periodistas en la sociedad $^{4}$.

Por último, la actitud se puede referir al carácter positivo o negativo que se atribuye al objeto (valencia) y a la intensidad de esa valencia. Por ejemplo, la simple aceptación o rechazo hacia la promoción de valores e ideas (del periodista abogado). La intensidad, en cambio, se representa a través de un continuo que evalúa aspectos positivos y negativos al mismo tiempo.
Una de las críticas más usuales para quienes realizan investigaciones sobre las actitudes es que no siempre hay concordancia entre lo que piensa, hace y dice una persona. Esta contradicción se conoce como disonancia cognitiva y se refiere "a que el conocimiento, información, actitud o creencias que tienen un individuo son contradictorios. Cuando una persona experimenta disonancia cognitiva se modifica la relación entre las actitudes y las conductas" (Dubrin, 2003).

Un ejemplo de disonancia cognitiva es cuando el reportero acepta sobornos de un funcionario público pero cuando se le pregunta si está de acuerdo con esta práctica la rechaza tajantemente. De cualquier forma, es un riesgo que los investigadores tienen que aceptar, tomar en cuenta y tratar de reducirlo.

Por otro lado, pese a que son resultado de un proceso interno $y$ complejo, las actitudes también son productos de la socialización y, por lo tanto, son algo modificable. Es decir, están sujetas a cambio. Un reportero que promueva determinados valores en su labor periodística puede, en un periodo de tiempo determinado, cambiar su actitud por uno que solo transmita la mayor cantidad de información en menos tiempo. Pero el cambio de actitud es un proceso complejo y largo que a veces requiere de habilidades.

Las actitudes se manifiestan a través a tres tipos de respuestas: las cognitivas, las afectivas y las respuestas conductuales. 


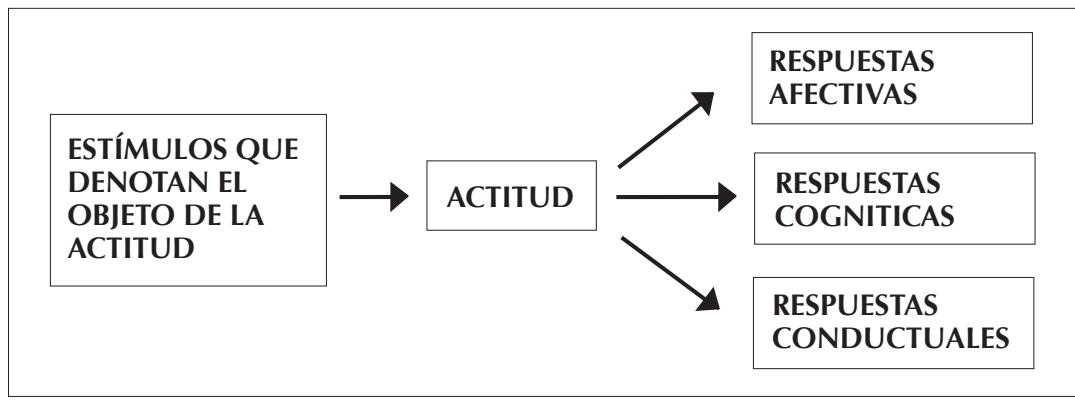

Fuente: Las tres respuestas a través de las que se manifiesta la actitud (tomado de Morales, Francisco; et al. Psicología Social, 1999, p. 195).

Las respuestas cognitivas se integran de percepciones, creencias, estereotipos, informaciones e ideas que posee la persona acerca del objeto. Las segundas se componen por los sentimientos que el objeto suscita en la persona. Por último, las respuestas conductuales que están compuestas por las tendencias, las disposiciones, las intenciones y las acciones que dirige hacia el propio objeto. Pero, ¿para qué conocer las actitudes?

Una razón es que nos permiten tener elementos para la predicción de conducta. Si un periodista se considera adversario del poder po- lítico podemos prever o inferir que esta persona establece una relación distante hacia la fuente que cubre o que sea más crítico respecto a la información que provenga de ésta.

$\mathrm{Y}$ por otro lado, no menos importante, es que las actitudes son la base de situaciones sociales muy importantes, como las relaciones de amistad y de conflicto. "Todo esfuerzo realizado por los sociólogos con objetivo de promover el bienestar humano, exige concentración sobre el papel desempeñado por las actitudes en la conducta humana" (Rodrigues, 1980).

\section{El cuestionario y sus destinatarios}

a elaboración de este artículo incluyó la revisión bibliográfica sobre modelos de comuni- cación y las actitudes profesionales de los periodistas. Además, se elaboró un cuestionario de 54 pregun- 
tas cerradas divididas en seis partes dirigido a los periodistas de política de cuatro medios impresos:

I. Características sociodemográficas

2. Condiciones laborales

3. Satisfacción

4. Experiencia

5. Prácticas profesionales

6. Actitudes profesionales

La selección de los reporteros, y por tanto de los periódicos, si hizo en base a los ciertos criterios. En primer lugar, que fueran periodistas que pertenecieran a un medio impreso de El Salvador, además que tuvieran asignada una o más fuentes políticas ${ }^{5} y$; por último, que el periódico no fuera de corte sensacionalista. Al final quedaron La Prensa Gráfica (LPG), El Diario de Hoy (EDH), Diario El Mundo (DEM) y el Diario Co Latino.

Si bien el periodismo político en El Salvador no se circunscribe a lo que hacen estos medios, en este artículo delimité la investigación a los medios que tuvieran características similares en su organización y forma de trabajo.

Aun así, hay diferencias importantes entre estos. Por ejemplo, respecto a la dimensión de las redacciones y la cantidad de periodistas que la componen.

Al momento de elaborar este artículo, las salas de redacción de La Prensa Gráfica y El Diario de Hoy estaban fragmentadas por áreas ${ }^{6}$ delimitadas entre si. Además existía una clara división del trabajo que se manifiesta en la pertenencia a una sección. Cada reportero tenía y tiene una o dos fuentes fijas asignadas. En cambio, en El Mundo y el Diario Co Latino las redacciones son pequeñas $y$, si bien hay reporteros encargados de las fuentes de política, la asignación está subordinada a las necesidades inmediatas de la redacción.

Sin embargo, durante una cobertura especial, en los cuatro periódicos por igual, se dispone de todos los periodistas de la redacción según lo amerite el caso. Por ejemplo, la emergencia que genera un terremoto. Los de política pueden cubrir una fuente no necesariamente política. De la misma forma, el día de las elecciones se sitúan reporteros de otras áreas para dar cobertura al evento, independientemente de que estos pertenezcan a otra sección.

En total, en el momento de la investigación había dieciocho periodistas destinados a la sección política en las cuatro redacciones: seis de La Prensa Gráfica, cinco de El Diario de Hoy, tres de El Mundo y cuatro de Co Latino. A cada uno de ellos se les explicó de manera personal en qué consistiría el cuestionario y todos, con excepción de dos reporteros de La Prensa Gráfica, accedieron a responderlo.

Debido a la naturaleza de la profesión periodística, que exige trabajar contrarreloj, algunos solicitaron responder al cuestionario al final de la jornada laboral y a otros se les tuvo que abordar durante una asignación. 


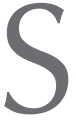
i describiéramos a grandes rasgos al periodista de política el perfil resultaría de la siguiente forma. Un hombre que no sobrepasa los 40 años, soltero y sin hijos. Inició sus estudios universitarios pero no los terminó. Pertenece a la sección de política de un periódico grande. Asegura sentirse satisfecho en su medio; sin embargo, dejaría el periódico para ganar más dinero $y$ en el futuro no se ve trabajando como periodista.

Este comunicador piensa que la razón de ser del periodista radica en su función social; y opina que su papel en la sociedad es investigar y explicar la información que afecte al público. Respecto al trato con la fuente considera que debe ser adversario del poder político; y para ello, debe cuestionar y dudar de aquella. En el plano ideológico, se ubica como alguien de centro.

Aunque encontramos cierta uniformidad en las características, los periodistas de política pueden manifestar actitudes opuestas entre ellos, incluso, en una misma persona.

\subsection{El perfil}

Los periodistas de política están separados en dos grupos a partir del año de nacimiento. De dieciséis entrevistados, seis nacieron entre 1960 y 1970 , mientras que los diez restantes lo hicieron entre 1970 y
1980. Pese a que la distribución marca una brecha generacional entre los dos grupos, se trata de un conjunto que en promedio no pasa los 36 años.

Al comparar este dato en otros países el resultado no cambia mucho. La edad promedio de los periodistas españoles y los estadounidenses es de 34 y 36 años $^{7}$ respectivamente, un poco menor a la de los salvadoreños.

Otro caso más próximo sucede con los reporteros de Colombia. De acuerdo a una encuesta realizada durante el 2003, por la Pontificia Universidad Javeriana de Bogotá, el promedio de los periodistas colombianos es de apenas 35 años ${ }^{8}$.

En resumen, de la misma forma que en otros países, los comunicadores de política salvadoreños son predominantemente personas que no sobrepasan los 40 años. Sin embargo, como se abordará más adelante, se trata de profesionales que pese a su corta edad cuentan con experiencia laboral, especialmente en área de política.

Los resultados además arrojan que las mujeres son la minoría: sólo cinco trabajaban en el área de política. Es posible que esto se deba a los roles que tradicionalmente le han sido designados a las mujeres. Por ejemplo, las responsabilidades que surgen al formar una familia. El 
dilema surge cuando la periodista asume sola el cuidado de sus hijos, del hogar y continúan con el ritmo de vida que demanda la profesión. Muchas veces, la periodista se ve obligada a renunciar. En España, por ejemplo, un buen número de mujeres se retira del periodismo antes de los 40 años, para dedicarse a tareas más compatibles con la familia.

Por otro lado, existe la creencia de que la política es cuestión de hombres, según asegura una periodista de El Diario de Hoy. "Las mujeres tenemos que hacer un es- fuerzo doble para que nos tomen en cuenta. Sucede que los editores son hombres; entonces tienden a pensar que sólo son ellos los que tienen la capacidad de meterse en el ruedo de la política. Donde yo trabajo ahora hay mitad y mitad (mismo número de mujeres y hombres) pero cuando son coberturas fuertes no mandan a una mujer. Por ejemplo, fue una mujer la que llevó el caso del diputado suplente Roberto Silva. Sin embargo, mandaron a un hombre a cubrir la culminación (El Diario de Hoy, nota publicada el jueves 30 de noviembre)".

\section{Figura 2}

Porcentaje de mujeres y hombres trabajando en política

\section{Mayor presencia de periodistas hombres}

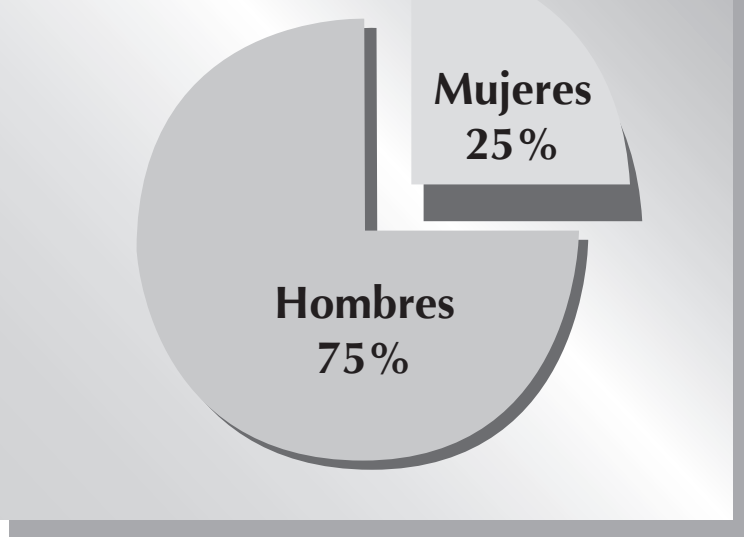

Fuente: Elaboración propia 
Respecto a su núcleo familiar, siete comunicadores están solteros, cuatro se encuentran acompañados y sólo cinco son casados; y de estos últimos, la mayoría tiene hijos. Es muy posible que en este caso también se repita el dilema que plantea dedicarse a un trabajo que no permite mucho espacio para la vida personal. Un periodista de El Diario de Hoy lo resume de la siguiente forma: "Cuando entrás al periodismo, te das cuenta de que perdés tu vida privada, porque tenés que estar disponible en vacaciones y los fines de semana. No te queda nada de tiempo para tu vida privada".

En el terreno profesional, de dieciséis entrevistados, siete son graduados universitarios y nueve iniciaron sus estudios pero no concluyeron su formación ${ }^{9}$. De los titulados, únicamente dos continuaron con estudios de postgrado. ¿Cuál es la razón de que hay pocos comunicadores con estudios de pregrado concluidos $y$, mucho menos, con postgrados?

Dada las características de la profesión, si bien el periodismo requiere de herramientas teóricas para su ejercicio, también es cierto que la profesionalización no depende sólo de esta condición. Más bien, el contacto con la sala de redacción y la práctica tienen una fuerte carga que determina en gran medida el éxito. Los periodistas, a diferencia de otros profesionales, pueden iniciarse sin haber terminado su carrera como parece ser el caso de los entrevistados.
Sin embargo, existen otros motivos que también influyen y que tienen que ver con la percepción de que no hay incentivos en una especialización. "Uno piensa que no sirve de nada tanto estudio porque los medios pueden poner a cualquier periodista redactor en esa área, que haga de todo, sin ser experto. Además, el que te especialices no significa que serás mejor pagado", asegura un comunicador de La Prensa Gráfica.

La posibilidad de ser sustituidos por un reportero más joven y con menos exigencias salariales los Ileva a acomodarse, aunque signifique abandonar sus estudios o no priorizar tiempo para especializarse en un área. Al momento de realizar la entrevista, sólo dos periodistas de $E I$ Diario de Hoy habían continuado con estudios de postgrado. El primero realizó estudios de periodismo en España y una segunda persona es egresada de la Maestría en Ciencia Política de la UCA.

Por otro lado, se les preguntó sobre condiciones laborales en las que trabajan. Específicamente, si tienen un contrato de exclusivi$\operatorname{dad}^{10}$. Sólo nueve respondieron afirmativamente. Aunque el resto no ha firmado esta estipulación su trabajo se limita a los periódicos que los contrató.

En El Salvador no es común que los reporteros tengan varios empleos al mismo tiempo; pero en otros países se trata de una práctica común ${ }^{11}$. Una de las maneras es compatibili- 
zar su labor periodística con tareas afines, como por ejemplo la cátedra. Esto permite adaptar los bajos salarios que siempre han caracterizado a la profesión periodística. De hecho, la remuneración es un tema que siempre causa dolor de cabeza entre los comunicadores.

Los medios tienen una política de apertura a jóvenes estudiantes, pero a veces esto va en detrimento del salario. Año con año, las salas de redacción reciben a practicantes universitarios que aceptan trabajar por bajos salarios, e incluso, sin siquiera recibir remuneración. En Bolivia, por ejemplo, un 16,6 por ciento de los periodistas tiene menos de 25 años y de estos la mayoría reciben bajos salarios. "Un 46,4 por ciento de los periodistas gana 2.000 bolivianos (305 dólares) o menos, lo que quiere decir que tiene ingresos similares a los segmentos de no profesionales. Solamente el 29,3 por ciento del total de los periodistas gana 3.000 bolivianos (460 dólares) o más. Uno de cada diez periodistas gana más de 5.000 bolivianos (770 dólares) mientras los periodistas de radio y las mujeres son los que menos ganan"12.

Los bajos salarios se vuelven el talón de Aquiles porque al Ilegar a cierta edad, pese a la experiencia acumulada, muchos periodistas dejan el medio para dedicarse a otras labores ligadas a la comunicación pero mejor pagadas.

\subsection{Remuneración y satis- facción laboral}

Si bien la satisfacción laboral depende de una serie de circunstancias como el reconocimiento, beneficios, condiciones del trabajo, relaciones laborales con los colegas; en este caso la mayoría de los reporteros la relacionaron con el salario que reciben. Durante las entrevistas la mayoría aseguró que renunciarían al periódico en el que trabajan para ganar más dinero, pese a que antes casi todos habían mostrado cierto grado de satisfacción laboral.

Se les pidió que ubicaran su opinión en una escala del 1 al 6, en donde 1 significa muy insatisfecho y 6 muy satisfecho. Siete se mostraron muy satisfechos de trabajar en su actual periódico. Igual cantidad de comunicadores ubican en el centro, entre el 3 y 4 . Únicamente dos expresaron que estaban muy insatisfechos.

A continuación se les abordó respecto al motivo por el cuál dejarían su actual trabajo. La mayoría, 7 en total, respondieron que renunciarían al periódico para recibir una mejor remuneración. En segundo lugar indicaron que dejarían su actual trabajo para disminuir el estrés y por frustración. Otros motivos menos recurrentes fueron crecer profesionalmente, desarrollarse académicamente y ampliar los conocimientos. 


\section{Figura 3}

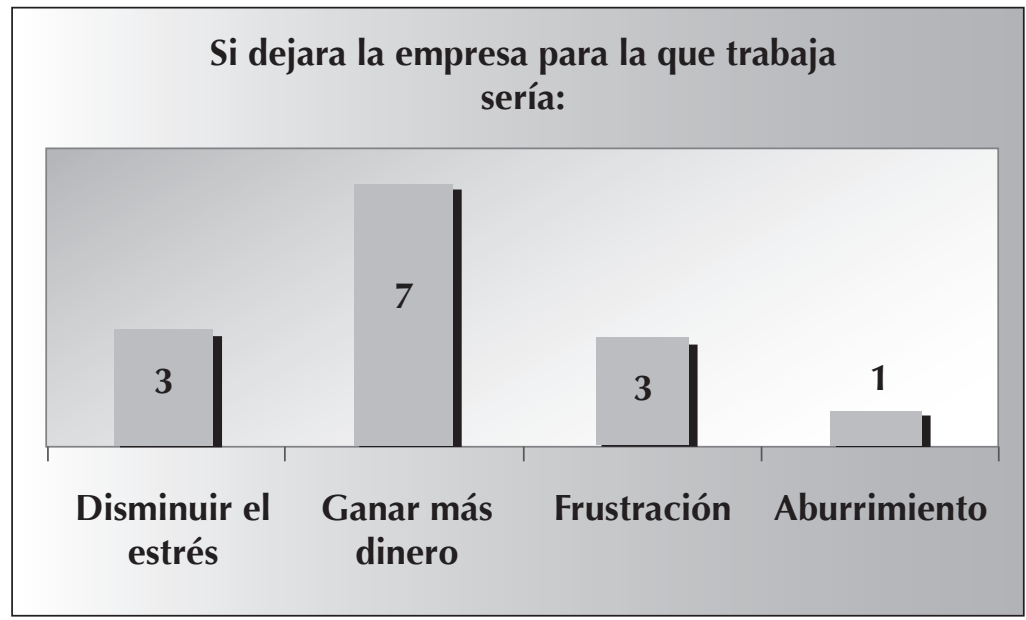

Fuente: Elaboración propia

"La gente no se siente satisfecha con lo que le están pagando, entonces casi siempre ven al periodismo como algo para mientras consiguen un trabajo en el gobierno, pongo mi negocio, me sale trabajo en una publicidad o cosas por el estilo. Esa es una aspiración de muchos estudiantes que comienzan a trabajar en los periódicos, al menos cuando yo era estudiante hace diez años", explica un reportero de El Diario de Hoy.

Otro periodista del Co Latino da las mismas razones. "Si haces una evaluación de la situación salarial de los periodistas, es bastante baja. Normalmente la gente hace trabajo periodístico porque le gusta, pero no por remuneración. Entonces, la gente dice 'bueno, me voy a sacri- ficar un tiempo en esto y luego voy a buscar un trabajo que me dé más ingresos'". ¿Influye el salario en la satisfacción laboral?

"Si tienes una buena remuneración, tienes más interés en hacer bien tu trabajo, porque tu familia está más estable y te da tranquilidad. En caso contrario, estás pensando en otras opciones que te generen ingresos", asegura un comunicador de La Prensa Gráfica.

De los dieciséis entrevistados, sólo uno aseguró sentirse más o menos satisfecho con su salario. "Ni mal y bien pagado", aseguró el periodista. Sin embargo, reparó en que, de acuerdo a su experiencia y sus estudios de postgrado en periodismo, lo ideal sería ganar el 30 por 


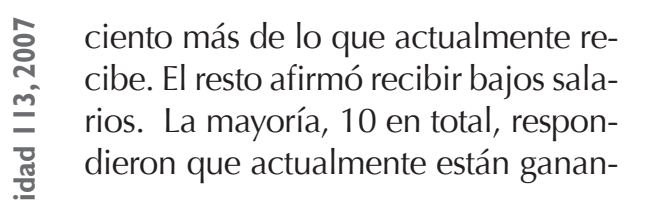

do menos de 650 dólares. La mitad de estos recibe menos de 450 dólares al mes. Únicamente tres periodistas aseguraron ganar más 851 dólares.

\section{Cuadro 1}

\begin{tabular}{c|c|c}
\hline \multicolumn{3}{c}{ Nivel de ingresos al mes } \\
\hline Rango & Frecuencia & $\%$ \\
\hline 450 o menos & 5 & 31.25 \\
\hline De 451 a 650 dólares & 5 & 31.25 \\
\hline De 651 a 850 dólares & 3 & 18.75 \\
\hline 851 dólares o más & 3 & 18.75 \\
\hline Total & 16 & 100 \\
\hline
\end{tabular}

Fuente: Elaboración propia

"La gente piensa que porque uno trabaja en un periódico importante y grande tiene un salario igual. ¡Uno ve al presentador de televisión bien bonito con su traje pero eso no significa que tiene buen salario! A veces es el que menos gana".

La variable salario también influye en que la mayoría no mostró interés por seguir trabajando en periodismo. Ante la pregunta ¿en dónde le gustaría estar trabajando en los próximos cinco años? Sólo seis señalaron que en un periódico; de estos, cinco dijeron que en donde se encuentran actualmente $y$ uno en un medio diferente. El resto, diez en total, no se ven trabajando en una empresa periodística. El poco interés que mostró la mayoría respecto a su futuro como periodistas apunta a que la profesión periodística no es en estos casos una opción laboral de largo plazo; más bien algo transitorio, como lo confirman durante las entrevistas a profundidad.

\subsection{Experiencia y prácticas laborales}

Respecto a su experiencia, casi todos señalaron haber trabajado en otro medio y, de estos, la mayoría en el área de política. Es decir, se trata de un grupo de reporteros con experiencia en el trato con la fuente política. 


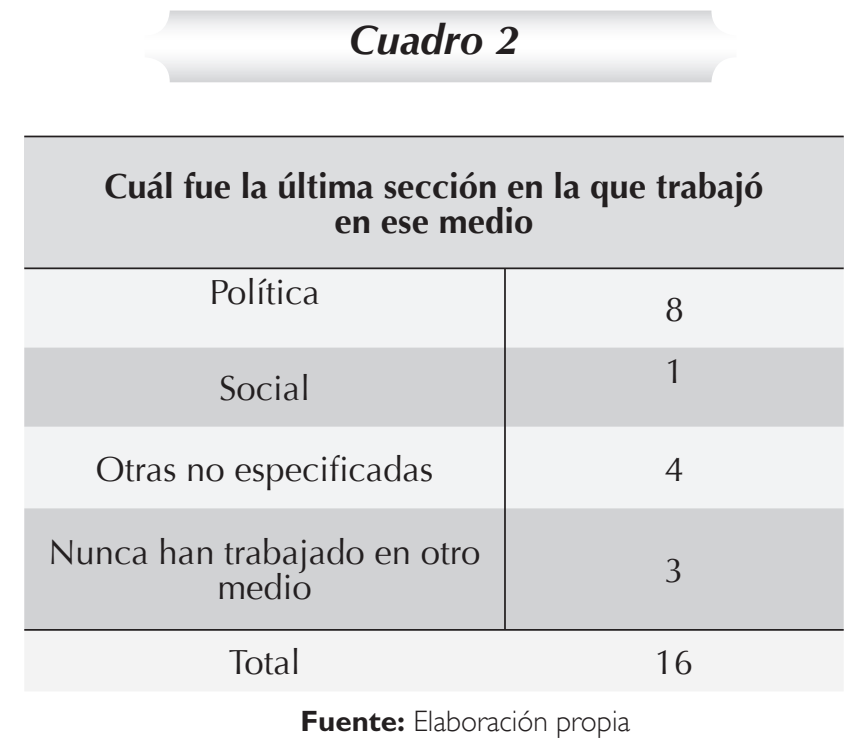

Para realizar su trabajo, los periodistas realizan una serie de prácticas que muchas veces pueden llegar a convertirse en vicios. Por ejemplo, el abuso del "off the record"13.

El "off the record" es un recurso que emplea el reportero para obtener información para su propio uso; aunque es publicable siempre y cuando sea confirmada o contrastada con otras entrevistas. Este recurso permite obtener información de fuentes que no quieren hablar en público por temor a represalias. Sin embargo, es indispensable que se establezca al inicio de la entrevista y a través de un acuerdo explicito entre la fuente y el periodista.

El problema surge cuando el periodista acostumbra a publicar sin referencias directas porque puede despertar dudas sobre la veraci-

dad de la nota publicada. ¿Es real la fuente o es una información publicada por el periodista con un propósito determinado? Y si fuera real la fuente, ¿con qué intensión da esa información?

Hay quienes recurren al "off the record" como última instancia para obtener una información, es decir sólo en casos excepcionales. Por otro lado, están los que hacen un uso indiscriminado de este recurso. Pero, ¿con qué frecuencia recurren a este tipo prácticas los reporteros salvadoreños de política?

Se les preguntó si recurren al "off the record" y quién suele decidir su uso. Catorce entrevistados respondieron que lo emplean frecuentemente. Respecto a quién lo decide, ocho aseguraron que es el periodista; seis, que la fuente $y$ 
dos, que es el editor. Si bien, esta información sólo describe de manera muy general el uso del "off the record", nos indica que se trata de una práctica común entre los comunicadores de política.

De igual manera, es frecuente que se publiquen notas sin crédito o nombre del autor, especialmente cuando se trata de temas controversiales. Un ejemplo es la entrega especial de La Prensa Gráfica en la que se denunció que altos dirigentes del FMLN son socios comerciales de Raúl Alexander Granillo, ex comandante de ese partido y que está actualmente acusado por secuestro (22 de noviembre, La Prensa Gráfica).

La entrega fue firmada sólo como "Redacción de Nación". En este caso, aunque haya una razón valida, de cara al lector únicamente se puede especular la razón por la que el periodista que investigó optó por no firmar esta nota.

El crédito en las notas es una práctica periodística que permite identificar al responsable de la nota ${ }^{14}$. Es decir, se trata de saber quien, en primera instancia, elaboró la nota y, por lo tanto, referirse como responsable de informa-ción publicada.

¿Qué tan frecuente es el uso de créditos entre los periodistas de po- lítica? Los resultados arrogaron que de dieciséis entrevistados, catorce consideraron válido no hacerlo. A los que respondieron de manera positiva se les dio cinco razones para que señalaran cuál de todas fue la razón por la que no firmaron la última vez: (1) el tema era polémico, (2) no estaba de acuerdo con la edición final, (3) había datos imprecisos en la nota, (4) peligraba mi integridad física y por último otra razón no señalada.

La mitad de los periodistas señalaron un motivo diferente a los dados. La razón más frecuente para no firmar una nota fue por que había sido reportada y escrita por diferentes personas. Otro motivo fue por ser una nota hecha a partir de un comunicado de prensa. En estos últimos casos, suele pasar que los periodistas sólo editan el comunicado y, a veces, contrastan la información oficial con otra fuente. Este ejercicio no representa mayor esfuerzo periodístico razón por la que el comunicador opta por no firmar esa nota.

En menor frecuencia, los entrevistados manifestaron que no firmaron por ser un tema polémico, que no estuvieron de acuerdo con la edición final de la nota, porque había datos imprecisos en la nota o que peligraba su vida. 


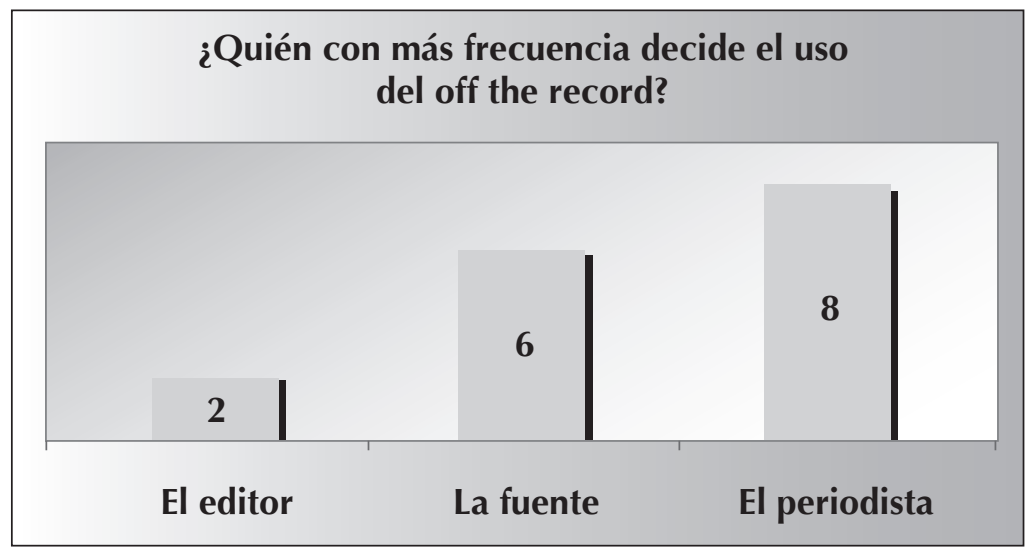

Fuente: Elaboración propia

\section{Actitud profesional del periodista}

E s común que sean los periodistas los que pregunten e indaguen sobre lo que piensan fuentes; pero qué sucede cuando en lugar de preguntar los comunicadores responden. ¿Qué opinan los periodistas en relación a su papel en la sociedad, a la fuente de información o de la información en si?

En esta parte vamos a comentar algunas posiciones que muestran los periodistas al expresar sus opiniones. Es decir, describiremos lo que en psicología se conoce como actitudes. Pero, ¿qué vamos a entender por actitudes profesionales de los periodistas?

Para abordar el tema de la actitud profesional respecto a la noticia y a la fuente política retomamos principalmente la tipología que María José Canel propone en su libro Comunicación Política; además, de otras propuestas hechas en los últimos años.

Johnstone, en 1976, plantea dos clasificaciones respecto a la noticia: el periodista es neutral o participativo. Esta tipificación parte del principio de objetividad que supone que el comunicador mantendrá una actitud distante ante a la información y sólo se limitará a trasladar íntegramente lo que ve. Pero el principio de objetividad entró en crisis en la década 1970 y, en los últimos años, ha ido perdiendo fuerza frente al "nuevo periodismo" que da valor a la subjetividad y el interés del reportero. 
Más adelante, en 1986 Weaver y Wilhoit objetaron la clasificación de neutral y participativo por considerarlos limitados. Los autores añadieron una tercera categoría: el adversario, el intérprete y el difusor. El periodista adversario considera que debe ser una especie de fiscalizador de los poderes y se distancia de las fuentes, especialmente la oficial. El intérprete cree que debe analizar la información que afecte al público. Por último, el difusor entiende que su papel es dar información al mayor número posible de personas en poco tiempo.

Diez años después, Canel, Sánchez Aranda y Rodríguez Andrés complementan la tipología: Estos autores proponen Ilamar analista al intérprete y agregan el periodista abogado. Este último, considera principalmente que su papel es promover valores.

Blumler y Gurevitch (Canel, 1999) plantean que el comunicador puede ser pragmático cuando evalúa y analiza la información independientemente de la fuente de donde provenga. En cambio, el periodista que muestre una actitud servil considera que la información oficial es importante para el público; por lo tanto, traslada todo lo que provenga de esta.

\section{1. ¿Analista?}

El periodismo como un ejercicio que conlleva responsabilidad social es una concepción que surge de una relación normativa entre los políticos y los periodistas. Es decir, "subyace una filosofía enemiga del poder y confiada en la libre información y en el debate de los asuntos públicos como medios para la educación política y para el autogobierno. Como consecuencia, la prensa, además de informar, entretener y vender, debe descubrir la verdad, y vigilar y controlar al poder políticos ${ }^{\prime 15}$. Pero ante la multiplicación de medios de comunicación y los abusos que se dan a partir del ejercicio de la libertad de prensa, en el siglo XX, surge la idea de adjudicarle cierta carga de responsabilidad social a la prensa. Pero ¿los periodistas salvadoreños se identifican con este compromiso?

Se les preguntó que tan de acuerdo están con la siguiente afirmación: "La razón de ser del periodista radica en su función social". Casi todos estuvieron de acuerdo o muy de acuerdo. Sólo uno se mostró en desacuerdo. Los comunicadores conciben que este compromiso se refiere a informar de la mejor forma posible. Aunque algunos se mostraron confusos en sus respuestas durante las entrevistas a profundidad, casi todos estuvieron de acuerdo en que el compromiso es informar de la mejor forma posible a sus lectores.

"Responsabilidad social, creo que es tratar de hacer lo más ético posible tu trabajo. Eso no es fácil porque tenés que tener una fuerte base académica. Tenés que saber muchas cosas y no dejarte engañar (por la fuente). Es intentar dar 
el mejor producto a costa de lo que sea. Tenés que olvidarte de tu yo, de tu familia y de todo para tratar de entregar el mejor producto". La explicación que da este periodista de El Diario de Hoy se puede contrastar con otra similar que da un colega del Co Latino. "Es necesario proporcionar a la población la información que necesita para tomar las decisiones correctas".

De la misma forma, un comunicador de La Prensa Gráfica asegura que además de informar es necesario orientar a los lectores. "En el sentido de hacer saber cómo están funcionando las cosas y cómo deberían funcionar. Por ejemplo, la responsabilidad de emitir el voto".

Estas creencias los llevan a identificarse, incluso, como defensores de la sociedad. "Si te fijas, hay dos superhéroes que son periodistas. Uno es Superman y el otro es Spiderman. Siento que tiene un poco de eso (la responsabilidad social)", asegura un periodista de El Diario de Hoy.

Otros piensan que este compromiso tiene límites. "Podés tener toda la buena intención de hacer tratamientos más completos y mejores de una realidad pero a veces hay limitantes que tienen que ver con la línea (editorial) de los periódicos. Entonces, hasta allí llega tu responsabilidad social".

En el sondeo también se les preguntó cuál consideran que es la función principal del periodista. La mayoría de los entrevistados opinan que es investigar y explicar la información que afecte al público. En general, de acuerdo con la tipología que propone Canel, los periodistas de política perciben que deben ser analistas de la política y suponen que su trabajo va más allá de ser simples mediadores del quehacer político. Además, suponen que las dos situaciones tienen relación directa con la actitud que deberían mostrar en su trabajo. "Significa que tenés que profundizar, contrastar todo lo que tu fuente te proporciona. Lo crítico tiene que ver con problematizar cualquier cosa porque detrás de un hecho qué es lo que hay. Sobre todo en nuestra realidad que las cosas no son como se muestran", explicó un periodista del Co Latino.

Esta idea presume que los reporteros son más que simples mediadores del quehacer político. Más bien, como se dijo con anterioridad, son los que en primera instancia deciden a qué darle más importancia y qué dejar en segundo plano. Desde la información más irrelevante hasta la más trascendental, casi siempre los reporteros tienen el poder de decidir qué y cómo hacerlo.

Por ejemplo, las coberturas periodísticas que hicieron de la sección plenaria en la que desaforaron al diputado suplente Roberto Silva acusado de lavado de dólares ${ }^{16}$. Si bien las cuatro notas coinciden en publicar un hecho común -el desafuero del diputado suplente Roberto Silva - cada periodista priorizó diferentes datos al inicio de la nota. 
En la publicada por La Prensa Gráfica, por ejemplo, prevalece el hecho de que "serán los tribunales de justicia los que decidirán si es culpable o inocente de blanquear millonarias cantidades de dólares...", mientras que en El Diario de Hoy sobresale el detalle de los únicos dos diputados que se abstuvieron de votar para desaforarlo: "Sólo Elizardo González Lovo, del PCN, y Guillermo Ávila Quehl, de ARENA, se abstuvieron de votar ayer para enviar a los tribunales al diputado suplente Roberto Silva. El primero por su amistad con el implicado, y el segundo, por ser el fiscal del antejuicio, al decidir no ser juez y parte en el proceso". En el caso del Co Latino se publica una nota en la que hay una mezcla de los dos primeros, sin embargo, más adelante en el cuerpo de la nota se dedica un espacio importante a contraponer declaraciones de las diferentes fracciones.

\section{Figura 5}

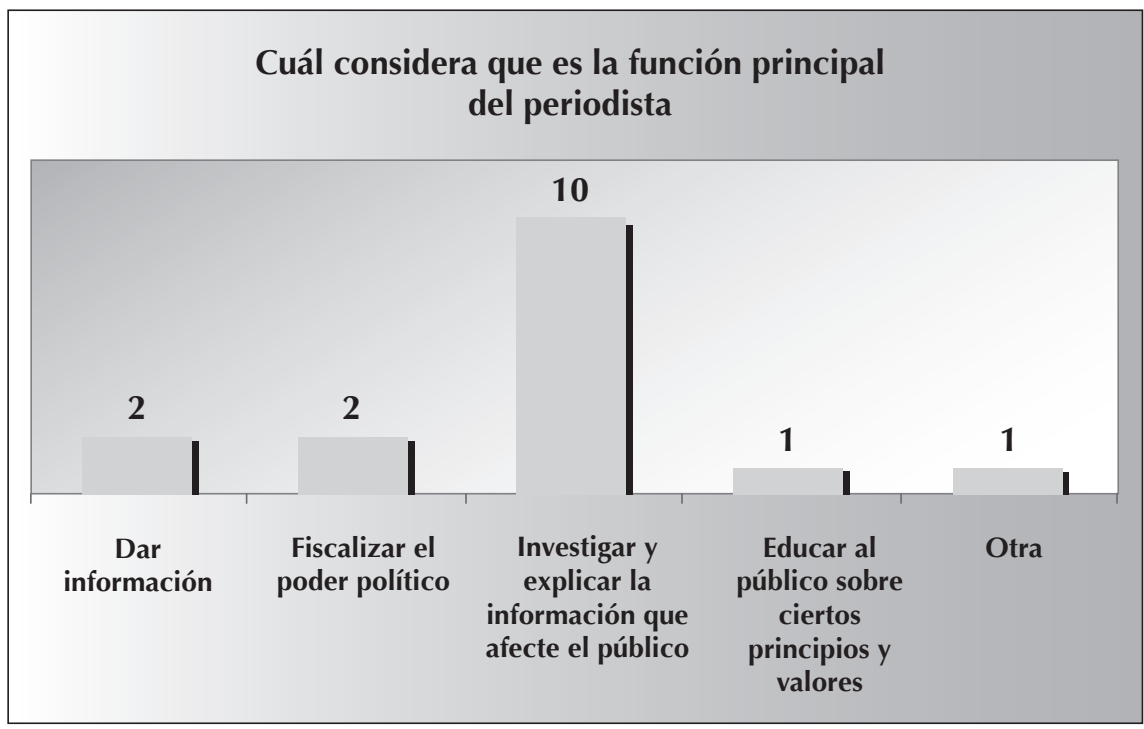

Fuente: Elaboración propia

\subsection{Dependencia entre perio- distas y fuente política}

La relación entre la fuente y los periodistas puede analizarse desde dos modelos. El primero es el adversarial, conocido como watchdog (perro guardián) que supone una relación de permanente conflicto. Concibe a los medios como defensores frente 
a los abusos del político ${ }^{17}$. El segundo modelo es el de intercambio que establece una relación de cooperación entre ambas partes. Es decir, los periodistas necesitan de sus fuentes para obtener información al mismo tiempo que las fuentes necesitan a los periodistas para posicionarse ante los potenciales votantes.

Para conocer la actitud que muestran al respecto los entrevistados se les preguntó qué tan de acuerdo está con la siguiente aseveración: "El periodista debe cuestionar y dudar siempre de la fuente". De los dieciséis periodistas, trece se mostraron muy de acuerdo. Esta actitud parece distanciarlos de su fuente. Sin embargo, durante la entrevista a profundidad matizaron este punto.

“El periodismo por definición debería ser adversario (de la fuente política) pero en la práctica te das cuenta de que no es así. Siempre hay que tener una relación de cordialidad, incluso de amistad, con la fuente. En definitiva son los que te dan la información. Dependiendo de cómo vos seas con la fuente, así te va a dar cosas buenas o churros ${ }^{18}$. Creo que eso se construye con el tiempo. Incluso, a veces les haces ciertos favores. ¡No hay que tomarlo a mal! Lo que pasa es que a veces un diputado te puede decir: 'mire, en mi comunidad hay problema de agua'. Uno va a la sección de Metro y les dice "Hey, mirá, saca está denuncia". Eso lo considera como un favor entre comillas", explica un reportero de El Diario de Hoy. El res- to de los comunicadores comparten la misma opinión. Pero, ¿ ¿hay límites en esta relación? Y si los hay, ¿cuáles son?

Durante las entrevistas a profundidad, todos coincidieron en que si existen límites y que se manifiesta cuando el reportero se vale de su trabajo para favorecer a la fuente. Por ejemplo, un reportero de $L a$ Prensa Gráfica asegura que si llega a pasar esto, terminarás informando para la fuente y no sobre la fuente. "Hay que tener cuidado cuando la relación es demasiado cercana, porque inhibe al periodista de hablar de la fuente".

De las opiniones vertidas en el sondeo y en las entrevistas surgen algunas preguntas obligadas que no podamos responder en esta investigación pero que al menos las plantearemos. ¿Los periodistas de política mantienen en realidad la distancia de la que hablan respecto a la fuente política? ¿Hay favores entre reporteros y fuente? Si los hay, ¿qué tipo de favores median en la práctica?

En el sondeo también se abordó la actitud del comunicador respecto a la información. Ante la pregunta "El periodista debe trasladar íntegramente lo que ve", trece comunicadores estuvieron muy de acuerdo. Esto supone que la mayoría se muestra a favor de comunicar tal y como sucedieron las cosas u objetivamente $^{19}$.

Con todo, esta actitud es empleada muchas veces como "ritual 
estratégico de defensa" (Tuchman, 1980). Es decir, para proteger su labor periodística de la crítica o la duda. De hecho, al preguntarles qué entienden por objetividad, todos estuvieron de acuerdo en que es equivalente a decir la verdad. Entonces, es de suponer que deben trasladar íntegramente lo que ven, de lo contrario creerían que están mintiendo.

Por último, al tomar decisiones, los periodistas deben elegir a veces entre formas alternativas de relato informativo. Si bien, la opción por uno $u$ otro a veces no depende de la voluntad del periodista también es cierto que existe un margen de maniobra importante para que decida como escribir su nota. Hay quienes intentan describir o contextualizar la información, mientras hay otros que se inclinan por exponer los hechos sin mayores matices. Ante la pregunta "¿cuál de los dos tipos de relatos suele adoptar?", catorce periodistas aseguran que sitúan los hechos en su contexto y sólo dos dejan hablar a los hechos.

\subsection{Los partidos políticos y la ubicación ideológica}

La tarea de gatekeeper supone, como se explicó en el apartado 2, una dimensión personal del periodista. Es decir, las creencias, opiniones y actitudes de la persona que pueden mediar durante la elaboración de una noticia. En ese sentido, es importante conocer también cómo perciben ideológicamente a los partidos políticos y en qué posición se ubican ellos mismos.

Se les pidió que ubicaran a los cinco partidos existentes-ARENA, FMLN, PCN, PDC y $\mathrm{CD}^{20}$ - en una escala del uno al diez, en donde 1 es la posición más a la izquierda y 10 más a la derecha ${ }^{21}$. Los resultados arrojaron que los partidos más grandes, ARENA y FMLN, fueron los más distantes entre sí. Del total, 14 periodistas ubicaron al primer partido entre 9 y 10 , mientras que el segundo entre 1 y 2 . El resto de los partidos políticos fueron situados en posiciones más o menos distantes a las extremas. Pero ¿qué significa la lejanía entre ARENA y FMLN? Principalmente que los periodistas tienen una percepción polarizada de los principales partidos políticos. Ahora bien, ¿influye esa idea en su labor periodística? Por el momento, podemos decir que es común, especialmente durante las campañas electorales, que los periódicos publiquen notas en las que se manifiesta una lucha cerrada entre dos partidos antagónicos ideológicamente y donde el resto no tiene cabida.

Los resultados también se pueden comparar con la autoubicación ideológica que hacen el conjunto de diputados en la misma escala. En el estudio sobre élites parlamentarias de América Latina que realiza desde 1994 el Instituto Interuniversitario de estudios sobre Iberoamérica y Portugal de la Universidad de Salamanca, España, rebela que existen una tendencia a que los di- 
putados también se ubiquen en posiciones extremas a los partidos más grandes. En el 2003, el FMLN salió posicionado en el 1.22 mientras que ARENA en 9.31 .

Por otro lado, se les pidió a los periodistas que se auto ubicaran en la misma escala. Es de señalar que esta pregunta causó incomodidad. Aunque se le recordó que el cuestionario era anónimo, algunos consideraron que su respuesta podría despertar sospechas de favoritismo hacia un determinado partido. Aunque accedieron a responder no podemos calcular que tan apegadas a la verdad fueron sus posiciones.

La mayoría, diez en total, se colocaron entre 5 y 6 ; mientras tres más en una posición de izquierda y centro izquierda. Los tres restantes en el centro derecha y derecha. Es muy posible que el predominio de la posición de centro esté más determinada por la idea de ser objetivo e imparcial que por su verdadera opinión.

\section{Ubicación ideológica}

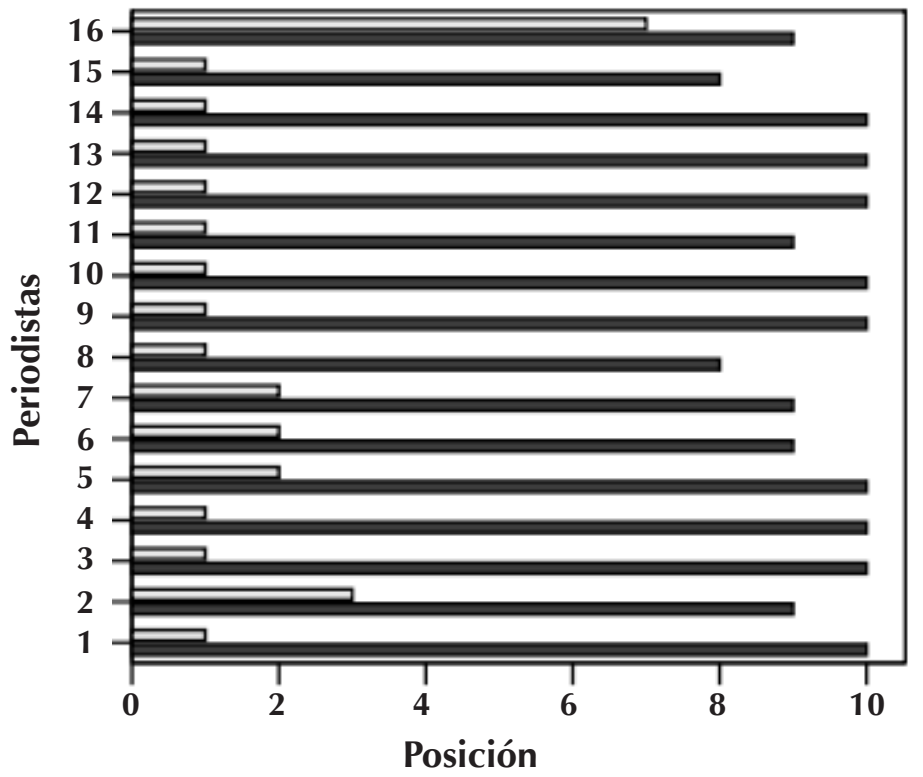

Ubicación

$\square$ ideológica de

ARENA
Ubicación

ideológica del

FMLN

Fuente: Elaboración propia 


\subsection{Los criterios de noticiabilidad}

¿Qué información política consideran importante los periodistas? ¿Por qué eligen de esa forma lo importante? En fin, ¿cuáles son los criterios de noticiabilidad que están mediando? Antes de responder es necesario definir que los criterios de noticiabilidad son en primer lugar "formulaciones pragmáticas, modalidades organizativas del trabajo cotidiano" (Martini, 2000).

Estos criterios además los vamos a entender como valores-noticias. "Los valores-noticia de un acontecimiento, son las cualidades significativas que constituyen su relevancia. La relevancia opera en el conjunto de periodistas de un medio, en el medio como empresa y en la sociedad" (Martín, 2000: 88).

Según Martín, los criterios de noticiabilidad se pueden clasificar según dos variables. La primera de acuerdo al efecto del acontecimiento sobre la sociedad. Es decir, se refieren a la novedad, originalidad, evolución futura de los acontecimientos, importancia, proximidad geográfica del hecho y a la magnitud por la cantidad de personas o lugares implicados. La segunda respecto a la comprensión de un acontecimiento, la credibilidad, la brevedad, la novedad y exclusividad del hecho entre otros.

Estos criterios son, por lo general, interiorizados y materializados, muchas veces de forma automática.
Sin embargo, existen otras razones que median de igual forma. Un criterio de noticiabilidad es el valor que el editor da a una determinada información. Por ejemplo, el editor o jefe de redacción consideran que es mejor un enfoque o darle prioridad a una noticia sobre otra. Si bien el periodista tiene un margen de maniobra para negociar o discutir, en caso que no esté de acuerdo con su jefe, en última instancia es el editor quien puede decidir. En la práctica, a veces estas razones suelen tener más peso.

Para conocer los criterios de noticiabilidad de los periodistas de política, se les solicitó que en una escala del 1 al 6 , en donde 1 significa poca y 6 mucha, que situaran la importancia de los siguientes criterios a la hora de determinar lo que publica su sección. A continuación las respuestas:

Sobre la importancia que tiene el juicio del jefe de redacción, doce periodistas respondieron 5 y 6 ; es decir, la mayoría piensa que el juicio del jefe de redacción es muy importante a la hora de determinar lo que se publica. Similar unanimidad hubo respecto al juicio del editor. Casi todos, 13 en total, dijeron que tiene mucha importancia.

En menor grado aparece lo que publica o emite la competencia o lo que indica el manual de estilo ${ }^{22}$. En el primer caso, 10 ubicaron en un punto medio esta razón. En el segundo caso, las respuestas fueron más variadas. Siete aseguran que tiene 
poca, 4 situaron en un punto medio y 5 si le dan mucha importancia.

En resumen, lo que piense el jefe es determinante a la hora de decidir lo que se publica. Tanto la opinión del jefe de redacción como la del editor de la sección son los criterios de noticiabilidad que mayor importancia tienen para los periodistas de política.

A continuación, se les proporcionó cuatro opciones respecto a la noticia política para que seleccionaran cuál suelen emplear más seguido. Seis comunicadores indicaron que priorizaban un tema "conflictivo, con vencedor y vencido", y la misma cantidad señaló que fuera "una información que acerque a gobernados y gobernantes".

Estas opciones de respuestas se pueden comparar con la ubicación ideológica que hacen de los partidos más grandes como se vio arriba. En este caso, se puede inferir que si optan por temas polémicos, en los que haya vencedores y vencidos, es razonable que perciban como opuestos tanto al FMLN como a ARENA.

\begin{tabular}{|c|c|}
\hline \multicolumn{2}{|c}{ Criterios de noticiabilidad } \\
\hline Que sea un tema conflictivo con vencedor y vencidos & 6 \\
\hline Qué sea una información que venga de una autoridad pública & 1 \\
\hline Que asea una información lo poco que hace un funcionario & 3 \\
\hline Una información que acerque a gobernados y gobernantes & 6 \\
\hline Total & 16 \\
\hline
\end{tabular}

Fuente: Elaboración propia

\section{Relaciones interpersonales}

L

a sala de redacción de un periódico es un submundo en el que se manifiesta una cultura organizacional ${ }^{23}$ propia del mundo periodístico y las secciones que la conforman llegan a ser más que simples espacios de trabajo.
Buena parte de la vida del periodista transcurre en un escenario en donde enfrentan miedos, dudas, desafíos y satisfacciones. En ese mismo espacio también se establecen relaciones interpersonales que pueden mediar en el trabajo periodístico. 
Requejo Alemán ${ }^{24}$ explica que la iniciativa de los reporteros está condicionada a los endogrupos que pueda conformar en una redacción. "Estos colectivos sociales se constituyen y dinamizan por iniciativa individual de los propios reporteros, quienes comparten sus discusiones y dudas con agentes que indistintamente pueden conformar su ámbito de confianza. Al parecer lo crucial en estos asuntos es la relación afectiva y de confianza que los reporteros otorgan a cada uno de sus endogrupos, esto es lo que facultaría a cada profesional la posibilidad de tener o no iniciativa durante el desenvolvimiento de su trabajo". Requejo Alemán explica que las rutinas profesionales observadas no influyen tanto en las decisiones del trabajo de reporteo como "los diálogos mantenidos con personas afines a su forma de pensar o admiradas dentro de una redacción". ¿Por qué una buena relación entre compañeros ayuda a una mejor producción periodística?

"Creo que por la simple dinámica de este trabajo. Lo que ocurre a las nueve de la mañana ya no es valido a las cinco de la tarde en política. Diferente es un accidente, porque si el accidente es a las nueve de la mañana lo que puede variar es el número de muertos pero no las posturas de las fuentes. En política a las nueve de la mañana es una cosa y a las cinco de la tarde es otra. Entonces, una sola persona no puede estar cubriendo un mismo evento. Si no te apoyas en tus compañeros, estás perdido", asegura uno de los entrevistados.

En el mismo sentido, hay periodistas que piensan que la buena relación con sus compañeros comienza desde el momento en el que llegas a la sección. "La colaboración hace que trabajés más rápido. Si sos nuevo, tenés a la mano una guía de fuentes y aprendés rápido el lenguaje propio de la sección", explicó una periodista de El Diario de Hoy.

¿Cómo una actitud puede finalmente incidir en el trabajo? "Una buena relación favorece, porque podés compartir información y criterios. Hay confianza para criticarse mutuamente. Es más, la armonía influye en tu estabilidad psicológico y emotiva lo que se verá reflejado en el trabajo".

Un periodista colaborador es posible que facilite mejor el trabajo en equipo respecto a uno que tenga una actitud individualista. Por ejemplo, el colaborador puede que a pesar de tener asignado un tema haga espacio para discutir el enfoque de una nota con un colega. En este caso, aunque el trabajo en equipo ${ }^{25}$ no es una condición para una mejor producción periodística de la sección, sí es necesaria cierta actitud colaboradora entre los miembros pues facilita los espacios para la discusión de temas o posibles enfoques. Pero, ¿qué respondieron al respecto los periodistas consultados?

En primer lugar, todos los entrevistados estuvieron de acuerdo 
con que una buena relación entre compañeros hace más llevadero el estrés característico del periodismo. Sin embargo, ante la afirmación "la sala de redacción es un lugar exclusivamente de trabajo", ocho estuvieron muy de acuerdo, seis se ubicaron en el centro y dos dijeron estar muy en desacuerdo. Es decir, que si bien los reporteros están de acuerdo en que las relaciones interpersonales en la sección son positivas también creen que la sección a la que pertenecen no es un espacio para establecerlas.

Para saber cuál es la actitud de los periodistas de política respecto a sus compañeros se les formuló una serie de preguntas. En una escala del 1 al 6 , se les pidió que dijeran qué tan de acuerdo están con las siguientes afirmaciones:

- El periodista, siempre que pueda, debe estar dispuesto a colaborar con sus compañeros. Casi todos, 14 en total, estuvieron muy de acuerdo. Uno se ubicó en el centro y uno más está muy en desacuerdo.

- El periodista debe manejar discretamente la información; incluso en su sección. Siete están muy de acuerdo, cinco se ubicaron en el centro y los cuatro restantes señalaron estar muy en desacuerdo.

- Una buena relación entre compañeros de sección favorece a la producción periodística. Todos estuvieron muy de acuerdo.

Las respuestas marcan dos tendencias en los periodistas de política. Por un lado, todos están de acuerdo con que una buena relación entre compañeros de la sección favorece a la producción periodística, y de estos, la mayoría cree que es necesario colaborar con sus compañeros. Por otro lado, la mitad de los entrevistados piensan que tienen que ser discretos con el manejo de su información, incluso con sus propios compañeros de sección. En esa misma línea respondieron cuando se les preguntó con qué frecuencia pide la opinión al respecto de un enfoque o tema. Casi todos manifestaron que rara vez piden ayuda. Sólo cinco dijeron que muchas veces. Es decir, se muestran colaboradores hacia sus compañeros, pero reservados respecto a su trabajo.

Uno de los momentos más importantes de la jornada laboral es el cierre. Generalmente los periodistas tienen que redactar contra el tiempo y con el material recopilado, sea el idóneo o no. Es común que es en estos momentos en los que puede asaltar cualquier duda, pero no todos tienen la disposición a colaborar.

Se les preguntó si están de acuerdo con que el cierre puede generar tensión entre los compañeros de la sección. Casi todos, trece de ellos, respondieron de manera afirmativa. Asimismo se les preguntó que tan de acuerdo están con la siguiente afirmación: "La hora de cierre es para el trabajo individual; cada quien es responsable de su producción". Siete dijeron estar en desacuerdo, seis estuvieron de acuerdo y tres estuvieron muy de acuerdo. 
Aunque casi todos estuvieron de acuerdo en que la hora de cierre es un momento crítico en la jornada

\section{Comentarios finales}

L

os periodistas de política de los medios impresos en El Salvador admiten que su labor tiene una carga de responsabilidad social que se manifiesta en la mejor nota posible; es decir, con todos los elementos necesarios para informar mejor a los lectores. Sin embargo, admiten que ésta labor termina cuando interviene la línea editorial del medio para el que trabajan.

Respecto al trato con la fuente política, mantienen una relación cordial que se sustenta en un beneficio mutuo. Tanto los periodistas necesitan de los políticos para obtener la información que desean como los segundos necesitan de los primeros. No existe una frontera visible y universal respecto los límites de esta relación. Más bien, los entrevistados afirman que es importante identificar cuando el trato con la fuente podría estar interfiriendo en un desempeño periodístico ético. Pero, ¿qué tipo de relación mantienen en la práctica? La respuesta periodística, la actitud predominante durante este momento es al trabajo individual.

es difícil de atender, especialmente cuando se trata de un ejercicio que no se puede evaluar tan fácil a simple vista.

Otra de las características que es importante destacar es la percepción polarizada que tienen de los partidos más grandes — FMLN y ARENA- Ambos partidos resultan en las extremas del espectro políti$\mathrm{co}$, como opuestos ideológicamente. La pregunta que es necesario plantear a continuación es: ¿media esta percepción en la producción periodística?

Por último, se trata de un grupo que reconoce la importancia de un trabajo en equipo. La dinámica del trabajo y las condiciones que median durante las coberturas hacen posible que se cree una especie de ambiente solidario, al menos en cuanto a trabajo se refiere, aunque la información parece indicar una preferencia por el comportamiento individual.

\section{Referencias bibliográficas}

- Andrew J. DuBrin, Fundamentos de Comportamiento organizacional, Thomson, México, 2002.
- Angelo Kinicke- Robert Kreitner, Comportamiento organizacional, Conceptos, problemas y prácticas, McGraw Hill, 2002 
- Alsina, Miguel Rodrigo, La construcción de la noticia. Ediciones Paidós, España, 1993.

- Azofra, María José, Cuadernos Metodológicos, n. 26, Cuestionarios, Centro de Investigaciones Sociológicas, Montalbán, España, 1999.

- Canel, José María, Comunicación Política, Técnicas y estrategias para la sociedad de la información, Tecnos, España, 1999.

- Coca, César y Peñalva, José Luis, Modelos de los medios de comunicación social. Servicio Editorial de la Universidad del País Vasco, Bilbao, 1998.

- María Pilar Diezhabdino, Ofa Bezunartea y Cesar Coca, La elite de los periodistas, Servicio Editorial de la Universidad del País Vasco, Bilbao, 1994.

- Morales, J. Francisco, etal. Psicología Social, Mc Graw Hill, España, Madrid, Segunda edición, 1999.

- Ortega, Felix y Humanes, María Luisa, Algo más que periodistas, Sociología de una profesión, Ariel, S.A., Barcelona, Primera edición 2000.

Notas

1 Existen cuatro teorías tradicionales sobre la prensa: la teoría autoritaria sobre la prensa, la teoría liberal, la comunista y teoría de la responsabilidad social. Esta última, de las cuatro la más reciente, surge en 1947 con la formación de la Comisión sobre la Libertad de Prensa que dio
- Quiroz Palacios, Abraham, Actitudes y representaciones, Temas actuales de la Psicología Social, Benemérita Universidad Autónoma de Puebla, México, Primera edición, 2004.

- Requejo Alemán, José Luis, "La iniciativa del reportero en la sala de redacción", Sala de Prensa, número 64, Febrero, 2004.

- Rodrigues, Aroldo. Psicología social, Editorial Trillas, México, cuarta edición, 1980.

- Sánchez Noriega, José Luis, Crítica de la seducción mediática, Comunicación y cultura de masas en la opulencia informativa, Tecnos, España, 2 edición, 2002.

- Tójar, Luis García, "La redacción como institución total”, Revista de estudios de comunicación, número 17, Zer, 1998

- Urrea Cuéllar, Javier, "La producción de noticias en medios escritos y televisivos de El Salvador", en Revista de Ciencias Sociales y Humanidades (Realidad), 94, 2003.

- "Lo que es el periodismo", Revista Enfoques, número 373, La Prensa Gráfica, 2005.

lugar a un estudio para replantear nuevas funciones de los medios de comunicación.

2 "La producción de noticias en medios escritos y televisivos de El Salvador", en Revista de Ciencias Sociales y Humanidades (Realidad), 94, 2003. 


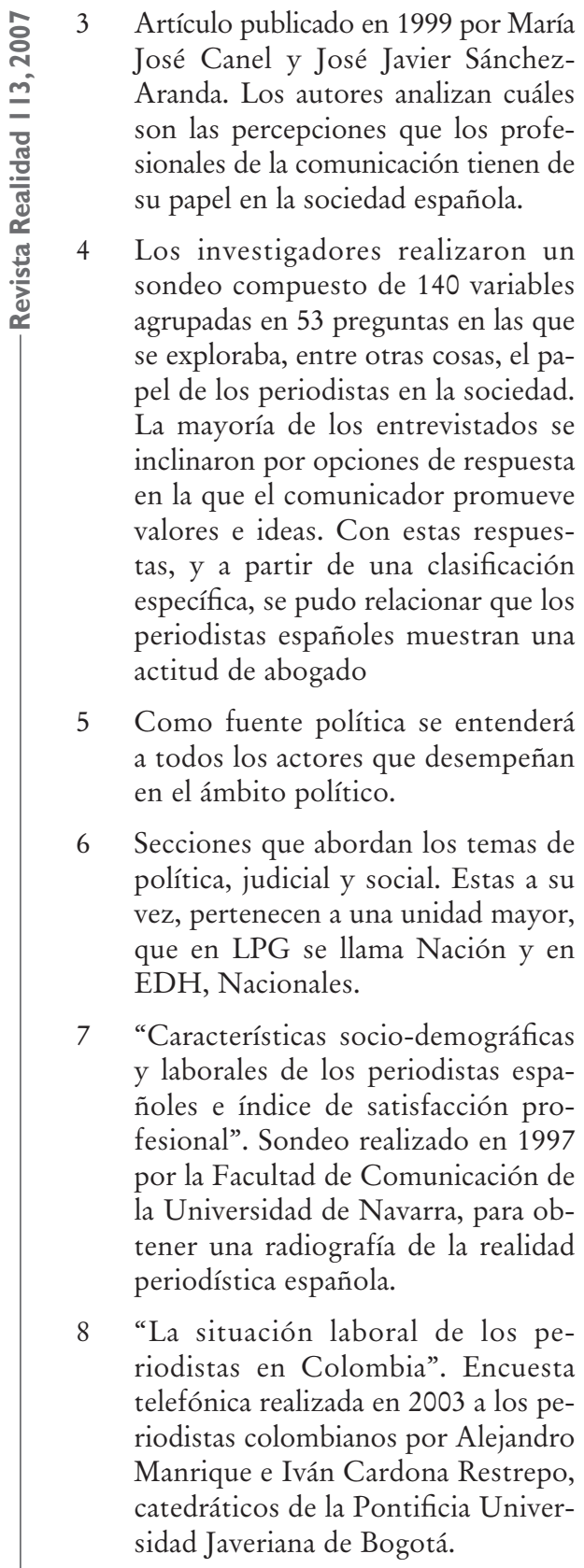

9 La Universidad de El Salvador (UES) fue durante muchos años la única institución educativa con una escuela de periodismo, fundada en 1955. En la década de los noventa, otras universidades abrieron carreras de periodismo. La Universidad Centroamericana José Simeón Cañas (UCA) fundó la licenciatura en Comunicación y Periodismo en 1990.

10 El contrato de exclusividad establece que todo lo que produzca el comunicador, durante el tiempo que determine el contrato, será propiedad del periódico. Esta condición puede incluir otras disposiciones más específicas.

11 Una investigación sobre la élite de los periodistas en España concluyó que más de la mitad de los profesionales entrevistados tienen otra actividad que les reportan ingreso.

12 Retrato del periodista boliviano, de Raúl Peñaranda Undurraga, es una investigación publicada en agosto del 2002 en la que se aborda el perfil del periodista boliviano.

13 "Off the record" es un término anglosajón que se refiere a que la información queda fuera de registro. Es decir, no se puede publicar la fuente que la proporcionó. Sin embargo, esta puede contrastarse con otras fuentes. El caso contrario es el "on the record" o "for the record" que significa que la información es para publicar e incluso se puede identificar a la fuente.

14 Si bien el contenido de la información es responsabilidad del periodista en primera instancia; una vez sea publicada el medio de comunicación es responsable por ella.

15 Canel explica que esta concepción proviene de una teoría liberal en la que se entiende que "no correspon- 
de al Estado controlar la calidad de la información sino que será el individuo quién, en el juego de la libre competencia, descubrirá cuál es la buena información".

16 Notas publicadas el 30 de noviembre en www.laprensa.com.sv, www. elsalvador.com, www.diariocolatino. com y en www.elmundo.com.sv de la plenaria extraordinaria en la que 82 diputados votaron para quitarle el fuero al diputado suplente Roberto Silva del Partido de Conciliación Nacional (PCN).

17 El modelo adversarial ha sido criticado, entre otras cosas, porque precisamente no toma en cuenta que los periodistas necesitan mantener cierta colaboración con sus fuentes de información para realizar su trabajo.

18 El término churro se refiere a información con poca transcendencia periodística.

19 El principio de objetividad entra en crisis en la década de los sesenta con el surgimiento del nuevo periodismo. Esta tendencia enfatiza la narrativa y la investigación, pero sobre todo la interpretación del propio periodista. Pese a las críticas que ha recibido este concepto, aun persiste la idea de un periodismo como ideal.

20 FMLN, Frente Farabundo Martí para la Liberación Nacional; PCN, Partido de Conciliación Nacional; ARENA, Alianza Republicana Nacionalista; PDC, Partido Demócrata Cristiano; CD, Cambio Democrático.
21 En la escala, las posiciones de la izquierda son 1 y 2, centro izquierda 3 y 4 , el centro 5 y 6 , el centro derecha 7 y 8 ; y por último, la derecha 9 y 10.

22 Usualmente los periódicos tienen un manual de estilo que sirve como organismo regulador de la política editorial, normas, criterios, estilos, etc. del medio de comunicación. En El Salvador no es común esta figura, sin embargo, hay disposiciones implícitas como por ejemplo la prioridad sobre temas y enfoques que responden a la política editorial del periódico.

23 Entenderemos cultura organizacional como "un sistema de valores y creencias compartidos que influyen en el comportamiento del personal. Las dimensiones de la cultura organizacional son los valores, las historias organizacionales que tienen significados subyacentes, los mitos, el grado de estabilidad, la distribución de recursos y premios, los ritos y rituales, el sentido de propiedad, y por último, espiritualismo corporativo y espiritualidad organizacional" (Dubrin: 278, 2003).

24 José Luis Requejo Alemán es doctor en Comunicación Pública por la Universidad de Navarra (España), especializado en procesos de producción de noticias (nerosmaking).

25 Trabajo en equipo visto como las personas que se proyectan su labor con un propósito común y se ven como un equipo. 